\title{
ARTICLE
}

\section{Transcriptional regulation of ethylene and jasmonate mediated defense response in apple (Malus domestica) root during Pythium ultimum infection}

\author{
Sungbong Shin ${ }^{1}$, Jingyi Lv ${ }^{3}$, Gennaro Fazio ${ }^{2}$, Mark Mazzola ${ }^{1}$ and Yanmin Zhu ${ }^{1}$
}

Apple replant disease (ARD) is a significant economic restraint to the successful re-establishment of new apple orchards on sites previously planted to the same crop. Pythium ultimum, an oomycete, is a significant component of the ARD pathogen complex. Although ethylene (ET)- and jasmonic acid (JA)-mediated defense responses are intensively studied in the foliar pathosystem, the transferability of this knowledge to the interaction between a perennial root system and soilborne pathogens is unknown. The aim of this study was to test the hypothesis that the ET/JA-mediated defense response is conserved in roots of tree crops in response to infection by $P$. ultimum. Apple genes with the annotated function of ET/JA biosynthesis, MdERF (ethylene response factor) for signaling transduction and a gene encoding a pathogenesis-related (PR) protein ( $\beta$-chitinase, the target of ERF) were identified from the apple genome sequences. The transcriptional profiles of these genes during $P$. ultimum infection and after exogenous ET and/or JA treatment were characterized using qRT-PCR. Several genes showed a 10- to 60 -fold upregulation in apple root tissue $24-48 \mathrm{~h}$ post inoculation (hpi). Exogenous ET and JA treatment exhibited either a positive or negative influence on expression of ET or JA biosynthesis genes, depending upon gene isoforms and the tissue types, while the expression of MdERF and the PR protein encoding gene was upregulated by both ET and JA treatment. Our data are consistent with the hypothesis that ET/JA-mediated defense pathways are functional in the root system of perennial tree species defending soilborne pathogens.

Horticulture Research (2014) 1, 14053; doi:10.1038/hortres.2014.53; Published online: 29 October 2014

\section{INTRODUCTION}

Apple replant disease (ARD) occurs when young trees are planted on a site that has a previous history of apple or closely related species cultivation. Symptoms of the disease can range from a mild uneven growth of young trees to serious growth inhibition or death of young trees. In the absence of control, the effects of ARD are experienced over the entire lifetime of the orchard in the form of decreased fruit yields. The ARD pathogen complex consists of oomycetes including Pythium and Phytophthora and fungi such as Ilyonectria (previously Cylindrocarpon) and Rhizoctonia, and at times acting in concert with the lesion nematode Pratylenchus penetrans. ${ }^{1,2}$ Control of ARD has traditionally relied on pre-plant fumigation of orchard soils; however, future availability of these fumigants could be increasingly restrictive due to environmental concerns. Utilization of rootstock resistance as an important component in managing ARD has not been carefully examined to date, partly due to the complex disease etiology and the difficulty in phenotyping the resistance response. ${ }^{3}$

Molecular defense responses of plant roots to soilborne necrotrophic pathogens are mostly unknown, and very few studies using root as a model to examine plant-necrotroph interactions. ${ }^{4,5}$ Using the model plant systems and foliar pathogens, ethylene (ET)- and jasmonic acid (JA)-mediated signaling pathways have been demonstrated to be a major part of plant defense reactions against necrotrophic pathogens. ${ }^{6-12}$ However, the transferability of this knowledge from foliar to root tissue is unknown. ${ }^{8}$ The relationship between ET and JA can be antagonistic or synergistic based on different stress signals including pathogen infection. ${ }^{7}$ This complex cross-talk between the two hormonal signaling pathways has been shown to be essential to elicit the appropriate plant reaction in response to pathogen invasion. ${ }^{13-15}$ Both ET- and JA-mediated plant defense responses are merged by activating the expression of ERF (ethylene response factor) and $P R$ (pathogenesis-related) genes. ${ }^{15,16}$ Recent studies from Arabidopsis showed that ERF1, originally thought to have a role only in ethylene signaling, actually responds to both ethylene and JA signals and integrates both hormonal signals to respond to necrotrophic fungal pathogens. ${ }^{6,15,17,18}$ Activated by both hormones, ERF1 regulates plant defense-related genes that have a GCC box on their promoter. Those defense genes appear to function primarily in response to challenges by fungal pathogens and herbivores. ${ }^{15,19}$

To test the hypothesis that ET/JA-mediated defense response is conserved in both leaf and root tissue, particularly in the root system of perennial tree crops, the transcriptional profiles of genes within the biosynthesis and signaling pathways of ET and JA were studied using apple root tissues infected by Pythium ultimum, a component in the ARD pathogen complex. The candidate genes from several gene families were identified from the apple genome sequences. ${ }^{20}$ These genes included biosynthesis genes for ET (MdACS: 1-aminocyclopropane-1-carboxylic acid synthase) and JA (MdAOS: allene oxide synthase), ET/JA signal response transcription factor genes (MdERFs) and a pathogenesis-related (PR) gene (MdCHIB: $\beta$-chitinase, the target of ERF). The transcription regulation of these genes to exogenously applied ET and JA was also investigated.

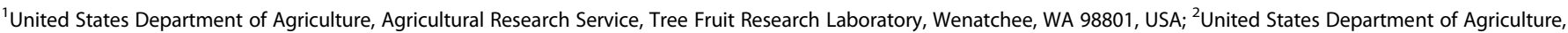
Agricultural Research Service, Plant Genetic Resources Unit, Geneva, NY 14456, USA and ${ }^{3}$ College of Horticulture, Northwest A\&F University, Yangling, shanxi 712100, China Correspondence: YM Zhu (yanmin.zhu@ars.usda.gov)

Received: 6 August 2014; Revised: 12 September 2014; Accepted: 17 September 2014
} 


\section{MATERIALS AND METHODS}

Preparation of apple rootstock seedlings and $P$. ultimum inoculum Seeds derived from a Geneva ${ }^{\circledR} 41(\mathrm{G} .41)^{21}$ and Malling 26 (M.26) ${ }^{22}$ cross were surface sterilized, germinated and planted in pasteurized Sunshine ${ }^{\mathrm{TM}}$ potting mix soil (SUN GRO Horticulture Ltd, Bellevue, WA, USA). Seedlings were grown at $23{ }^{\circ} \mathrm{C}, 95 \%$ humidity, under $12 / 12 \mathrm{~h}$ light/dark conditions for 3 weeks.

The $P$. ultimum isolate used in this study was originally isolated from the roots of 'Gala'/M26 apple grown at Moxee, WA, USA. ${ }^{1}$ Inoculum of $P$. ultimum was prepared by cultivating in potato carrot broth $(20 \mathrm{~g}$ of carrots and $20 \mathrm{~g}$ of potatoes in $1 \mathrm{~L}$ of medium) with two drops of wheat germ oil added per liter of medium. The $P$. ultimum cultures were grown in the broth in Petri dishes at $22{ }^{\circ} \mathrm{C}$ for 2-3 weeks. Oospores and mycelium were separated from the media by passing through a double layer of cheese cloth and the resultant mat was ground in a blender for $30 \mathrm{~s}$. Spores and hyphal fragments were resuspended in $2 \%$ methyl cellulose to give a final concentration of approximately 2000 colony forming units $\mathrm{mL}^{-1}$.

The inoculation of seedlings with $P$. ultimum was performed by dipping $70 \%-80 \%$ of the entire root system into the inoculum for $5 \mathrm{~s}$; then inoculated seedlings were planted into pasteurized Sunshine ${ }^{\mathrm{TM}}$ potting mix. The inoculated seedlings were grown in an environmental growth chamber at $23^{\circ} \mathrm{C}$, $95 \%$ humidity, under a 12/12 h light/dark photoperiod. Plants root tissues were collected at $0,2,4,8,24,48,72$ and $96 \mathrm{~h}$ post inoculation (hpi) and collected tissues were frozen in liquid nitrogen. Root tissue of twenty seedlings was collected and pooled for each time point per treatment. The experiment was repeated twice. Frozen root tissues were stored at $-80{ }^{\circ} \mathrm{C}$ until total RNA isolation. Un-inoculated control seedlings were dipped into $2 \%$ methyl cellulose solution and planted into pasteurized Sunshine ${ }^{\mathrm{TM}}$ potting mix.

\section{ET and JA treatment}

Seedlings were prepared as described above. Ethylene treatment was performed by purging 110 ppm ET (background with air) into a 3.8 L-size jar for $30 \mathrm{~s}$. JA treatment was performed by pouring $500 \mu \mathrm{M}$ methyl jasmonate (Sigma-Aldrich, St. Louis, MO, USA) in $0.177 \%$ Tween 20 into 2.25 inch square pots (Jiffy Products Ltd, Shippagan, Canada) until the pasteurized Sunshine ${ }^{T M}$ potting mix soil was saturated. The seedlings were treated at room temperature for $6 \mathrm{~h}$ at which time plants were extracted from the potting soil. Root and shoot tissue of 15-20 seedlings were collected and pooled per treatment. The experiment was repeated twice. Roots were excised from shoots and separately placed in liquid nitrogen. Frozen tissues were stored at $-80{ }^{\circ} \mathrm{C}$.

Primer design and phylogenetic tree construction Forward and reverse primers for MdACSs, MdAOSs, MdERFs, MdCHIB and MdActin genes (Table 1) were designed using web-based Primer3plus software (http://www.bioinformatics.nl/cgi-bin/primer3plus/primer3plus.cgi) and an IDT oligo analyzer (http://www.idtdna.com/analyzer/Applications/
OligoAnalyzer/). Where possible, an optimum annealing temperature of $60{ }^{\circ} \mathrm{C}$, a GC content of $40 \%-60 \%$, an amplicon length of $150-180 \mathrm{bp}$ and a primer length of $20 \mathrm{bp}$ were applied. Multisequence alignment software ClustalW (http://www.ebi.ac.uk/clustalw) and BLAST (http://www.ncbi.nlm .nih.gov/BLAST/) were used to choose divergent regions at or close to $3^{\prime}$ untranslated region for gene-specific primer design. Amplicons were sequenced to confirm identity of the specific amplified genes. Clustal W2 from EMBL-EBI (http://www.ebi.ac.uk/embl/) website and the Jalview 2.6.1 program were used to identify similarities between amino-acid sequences and to construct a phylogenetic tree. To calculate the distance between sequences, BLOSUM62 was used. Neighbor joining method was used for the phylogenetic tree construction.

RNA isolation and real-time qRT-PCR

The frozen tissue samples were ground to a fine powder in liquid nitrogen and total RNA was isolated following the modified method described by Gasic et al. ${ }^{23}$ The total RNA was quantified using a ND 1000 Nanodrop spectrophotometer (NanoDrop Technologies, Wilmington, DE, USA) and the RNA quality was verified by agarose gel electrophoresis. Total RNA was treated with DNase I (Qiagen, Valencia, CA, USA) and then purified with RNeasy cleanup columns (Qiagen, Valencia, CA USA). Two micrograms of DNase-treated RNA was used to synthesize first-strand CDNA using SuperScript ${ }^{\mathrm{TM}}$ II reverse transcriptase (Invitrogen, Grand Island, NY, USA) and poly dT (Operon, Huntsville, AL, USA) as the primer. The cDNA was diluted 20 times and $0.6 \mu \mathrm{L}$ aliquot was used in a $15 \mu \mathrm{L}$ quantitative PCR (qPCR) reaction mix: $0.45 \mu \mathrm{L}$ SYBR Green I dye (Invitrogen, Grand Island, NY, USA), 1X iTaq buffer (Bio-Rad, Hercules, CA, USA), $0.2 \mathrm{mM}$ dNTP (Applied Biosystem, Foster City, CA, USA), $2.5 \mathrm{mM} \mathrm{MgCl} 2,0.3$ units of iTaq DNA polymerase (Bio-Rad, Hercules, CA, USA) and $0.2 \mu \mathrm{M}$ forward/ reverse primer (IDT). Real-time GPCR amplification and detection was conducted using an iQ5 real-time qPCR detection system (Bio-Rad Lab, Hercules, CA) and the following protocol: cycle conditions of $3 \mathrm{~min}$ at $95{ }^{\circ} \mathrm{C}, 40$ cycles of $10 \mathrm{~s}$ at $95{ }^{\circ} \mathrm{C}$ and $30 \mathrm{~s}$ at $59{ }^{\circ} \mathrm{C}$. Dissociation curves were run for MdACSs, MdAOSs, MdERFs, MdCHIB and MdActin primers to determine the presence of any non-specific amplification. The relative gene expression was measured using the $0 \mathrm{~h}$ root tissue (for $P$. ultimum infection) or no-hormone treated control (for ET and JA transcriptional regulation) as the calibrator. 'No reverse transcriptase' and 'no template' negative controls were included in PCR amplification. Two sets of root samples (biological replicates) were used to obtain two independent total RNA sample sets, and subsequently converted into two separate cDNA sample sets. For each CDNA sample set, PCR reactions were performed twice. Therefore, four values of PCR amplifications (four data sets per sample: two biological replicate, each with two technical replicates) were performed from two independent biological samples. PCR amplification was carried out in triplicate in a 96-well plate. The target gene expression was normalized to that of the internal reference gene (MdActin) using the $2^{-\Delta \Delta \mathrm{Ct}}$ method (the comparative $C_{\mathrm{t}}$ method). ${ }^{24,25}$

Table 1 Selected genes and gene-specific primers

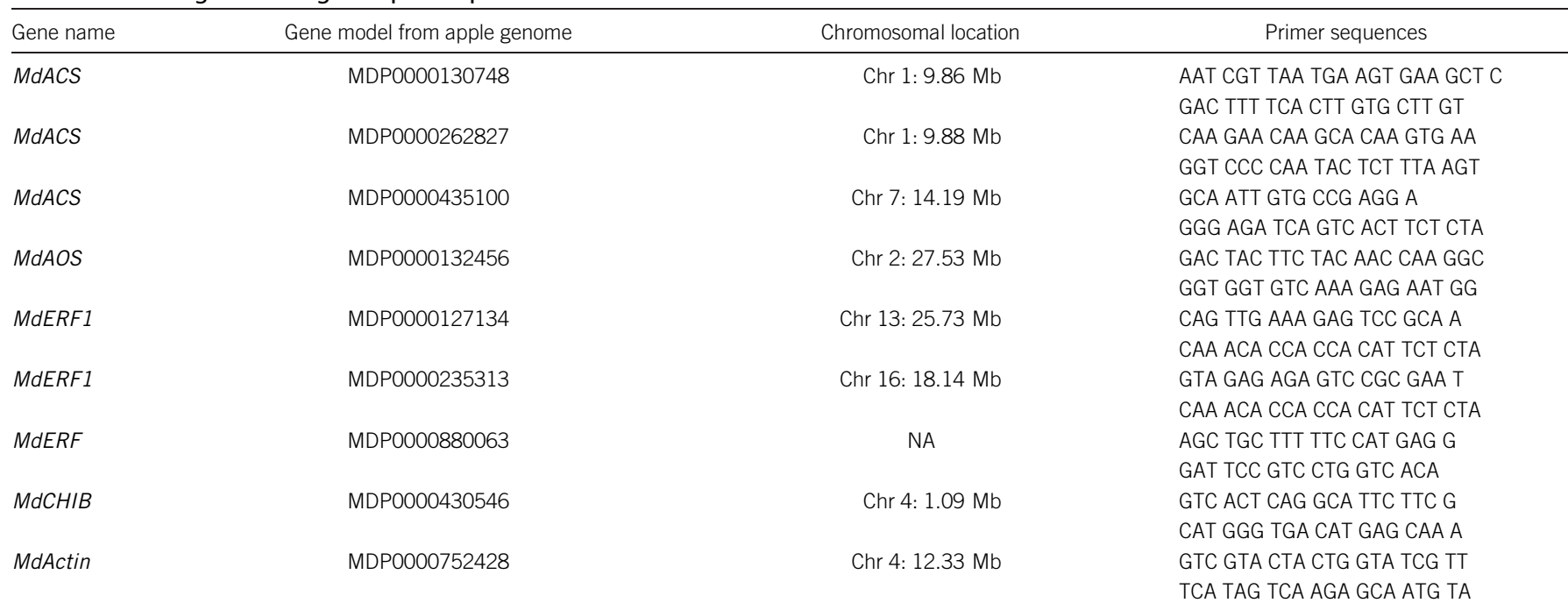




\section{RESULTS}

The apple root symptom from $P$. ultimum infection

A representative image of $P$. ultimum infected apple root tissues is shown in Figure 1. Compared with un-inoculated controls showing a white and healthy root tissue (Figure 1a), tissue necrosis with dark brown coloration throughout the root was typically observed on $P$. ultimum infected root (Figure 1b). It was also shown that the actively growing area of roots, especially the tips of emerging lateral roots or primary roots, are highly susceptible to infection by $P$. ultimum (Figure 1b). The symptoms represented in this figure were routinely observed.

Expression profiles of ET and JA biosynthesis genes in response to $P$. ultimum infection

To identify the members of the gene family for ET and JA biosynthesis that are induced in response to the $P$. ultimum infection, expression patterns of apple MdACS and MdAOS genes were examined. The phylogenetic tree for these fifteen MdACS genes was constructed by comparing their encoded peptide sequences and constructing a phylogenetic tree (Figure 2). The phylogenic analysis divided MdACSs into three subgroups: Groups I, II and III (Figure 2). Among the 15 MdACS isoforms, three genes (MDP0000130748, MDP0000262827 and MDP0000435100) exhibited a 6-60 time increase of transcript abundance within $48 \mathrm{hpi}$ of $P$. ultimum (Figure 3a-3c). The expression data of the MdACSs that were not affected by $P$. ultimum infection are provided in Supplementary Fig. S1. Among the three $P$. ultimum infection induced MdACS genes, MDP0000435100 showed the highest response at a time point $24 \mathrm{~h}$ after inoculation (Figure 3c). All three MdACS genes that responded to $P$. ultimum belong to the same sub-group (group I), while the well-studied fruit ripening related ethylene biosynthesis genes

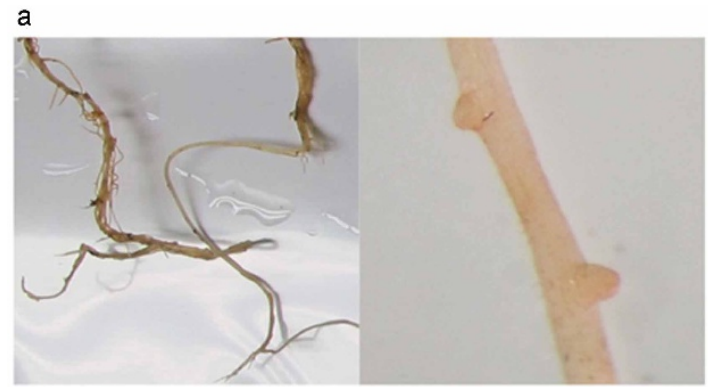

b

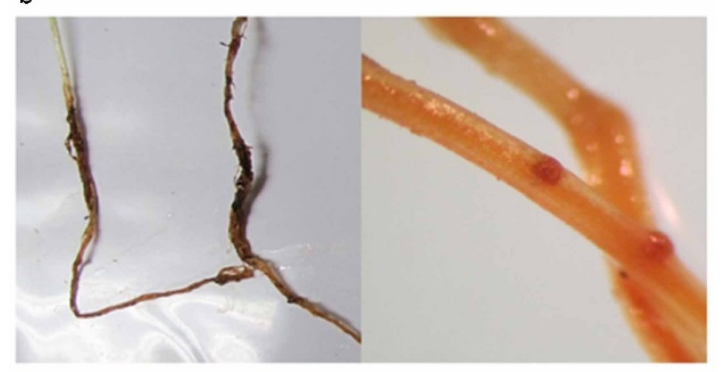

Figure 1. The observed symptoms of $P$. ultimum infected apple root tissue. (a) Un-inoculated control shows a healthy root tissue with light color. (b) P. ultimum-infected root show a dark brown necrosis. The close-up images on tips of emerging lateral root in un-infected control (a) and P. ultimum-infected root system (b) also demonstrate a difference in coloration between healthy tissue (a) and necrosis tissue (b).
(MdACS3 and MdACS1) belong to group II and III, respectively (Figure 2) and the expression of these fruit ripening-related genes was not affected by P. ultimum (Supplementary Fig. S1). Subgroups of the MdACS family and their differential gene expression in ripening and $P$. ultimum inoculation indicated that MdACS isoforms specifically participate in distinct plant processes.

Similarly, the expression profiles of members from the MdAOS gene family (MDP0000132456, MDP0000150140, MDP0000424398, MDP0000198152 and MDP0000225501) for JA biosynthesis in apple root tissue were investigated in response to inoculation with $P$. ultimum. Among the five genes examined, one MdAOS (MDP0000132456) showed strong upregulation in root tissues inoculated with $P$. ultimum (Figure 3d). Peak expression of this gene was observed 48 hpi with at least a 40-fold increase in transcript abundance, which corresponded with the expression pattern of the three MdACS genes (Figure 3).

\section{Identification of $P$. ultimum responsive MdERFs and MdCHIB}

Four ERF genes (MDP0000235313, MDP0000167207, MDP0000127134 and MDP0000880063) in apple showed an upregulation upon inoculation with $P$. ultimum (Figure 4a-4c). Based on genomic location and DNA/peptide sequence identity (data not shown), two of the MdERF1 genes (MDP0000167207 and MDP0000127134) were considered to be the same gene, and therefore only MDP0000127134 was included in further studies. Timing of the observed upregulation in the expression of the MdERF genes corresponded with the ET/JA hormone biosynthesis gene expression and was highly induced 24-48 hpi (Figures 3 and 4). Additional MdERFs that were not upregulated are described in Supplementary Fig. S3.

Bioinformatic analysis demonstrated that the upregulated apple MdERFs share a substantial similarity in peptide sequences with their homologous Arabidopsis AtERFs: MdERF1 (MDP0000235313 and MDP0000127134) possessed 55\%-56\% identity and 62\% similarity to AtERF1 (At3g23240); ${ }^{19}$ MdERF (MDP0000880862) had 43\% identity and $58 \%$ similarity to AtERF (At1g04370) ${ }^{26}$ (Figure 5a). These three MdERFs and two AtERFs showed a consensus AP2/ ERF (APETALA2/ET response factor) domain ${ }^{27}$ (Figure 5a). The phylogenetic tree indicated that MdERF1 (MDP0000235313 and MDP0000127134) is closely related to the well-characterized pathogen-responsive AtERF1 (At3g23240) ${ }^{19}$ and ORA59 (At1g06160) ${ }^{28}$ (Figure 5b).

An apple chitinase gene (MdCHIB: MDP0000430546) was also identified to be upregulated in response to infection by $P$. ultimum. CHIB (CHITINASEB) has been known to be a pathogenesis-related (PR) gene and its promoter region is a target of the ERF transcription factor. ${ }^{16,19,28}$ MdCHIB (MDP0000430546) exhibited a strong upregulation in response to $P$. ultimum infection, particularly in the period from 48 to 72 hpi (Figure $4 d$ ).

Transcriptional regulation of apple $P$. ultimum-activated genes to exogenous ET and JA treatment

To gain more insight into the ET/JA hormonal regulation of $P$. ultimum infection-activated genes, the expression patterns of MdACS, $M d A O S, M d E R F$ and MdCHIB in response to exogenous ET/JA treatment were studied. All three $M d E R F$ genes were upregulated by either ET or JA (Figure 6a-6f). Moreover, when ET and JA were applied in combination, a synergistic response at the gene expression for all MdERF genes (i.e. a higher fold changes) was observed (Figure 6a-6f). Although regulation of these two MdERF1 was similar, the expression of MDP0000235313 exhibited greater sensitivity to ethylene than did MDP0000127134 (Figure 6a-6d). Unlike the other two MdERFs, MDP0000880063 was induced to a higher level by JA than by ethylene (Figure 6e-6f). ET and JA appeared to act synergistically in the induction of the MdCHIB gene expression (Figure 6g-6h).

The expression of the three MdACS genes was regulated by ET and JA signals both antagonistically and synergistically. For two of 


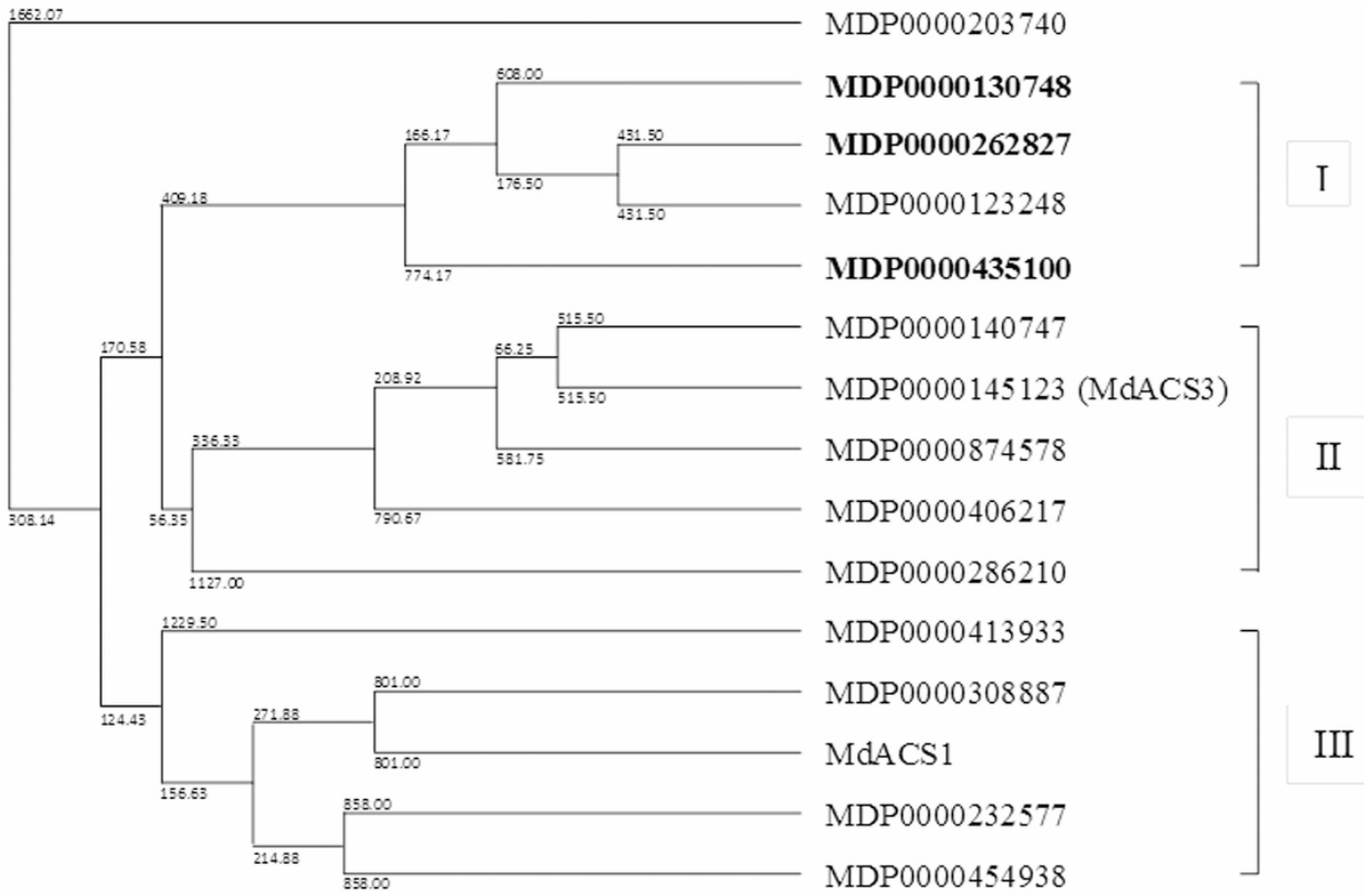

Figure 2. Phylogenetic tree of all apple 15 MdACS genes. Based on deduced amino-acid sequence similarity, MdACSs were divided into three groups: group I, II, and III. Three MdACSs that were upregulated by the P. ultimum infection are in bold. Md, Malus domestica.
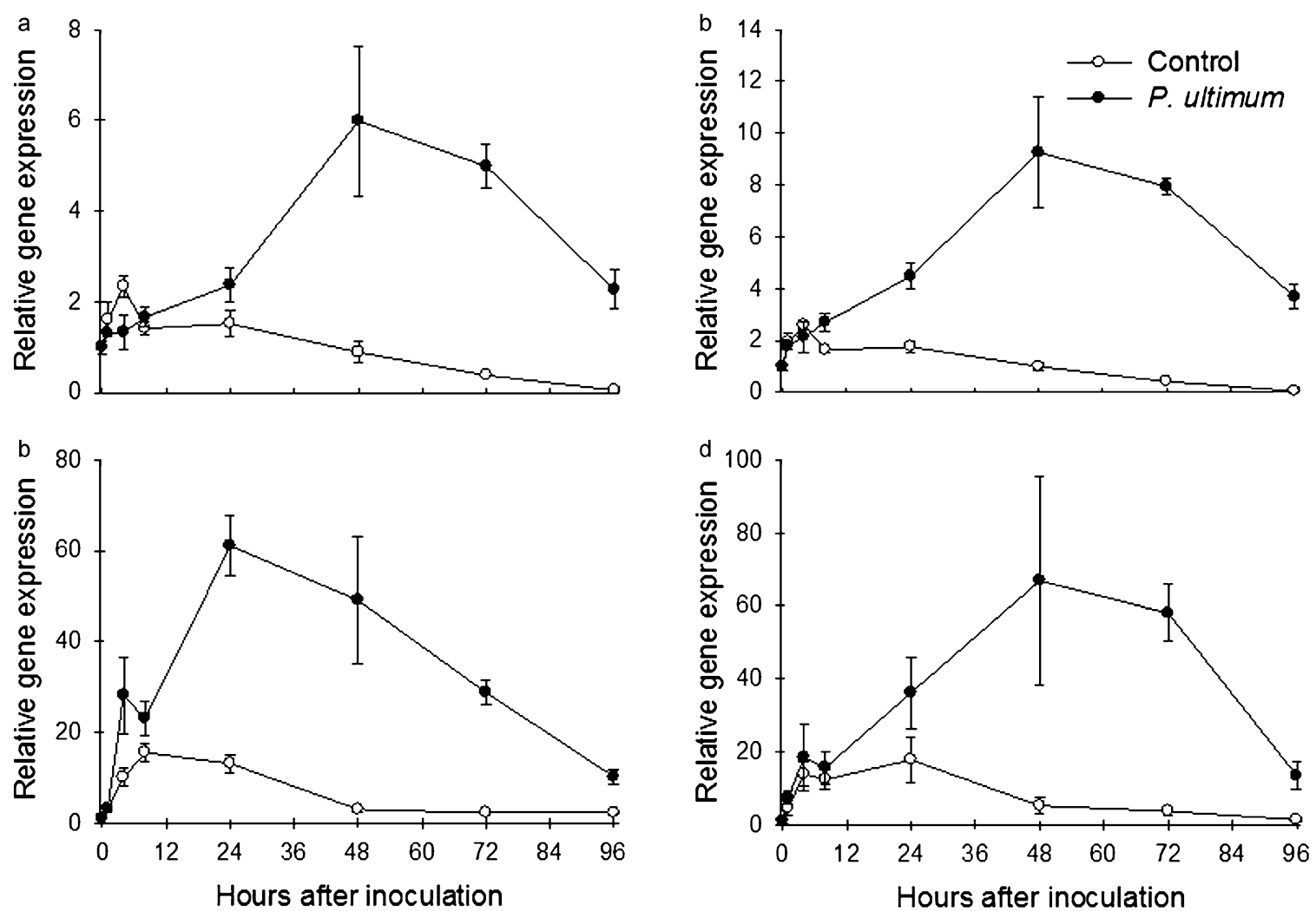

Figure 3. Gene expression profile of three apple MdACS and MdAOS in response to $P$. ultimum in apple root: (a) MdACS (MDP0000130748); (b) MdACS (MDP0000262827); (c) MdACS (MDP0000435100); (d) MdAOS (MDP0000132456). Each data point represents the means with standard error. 

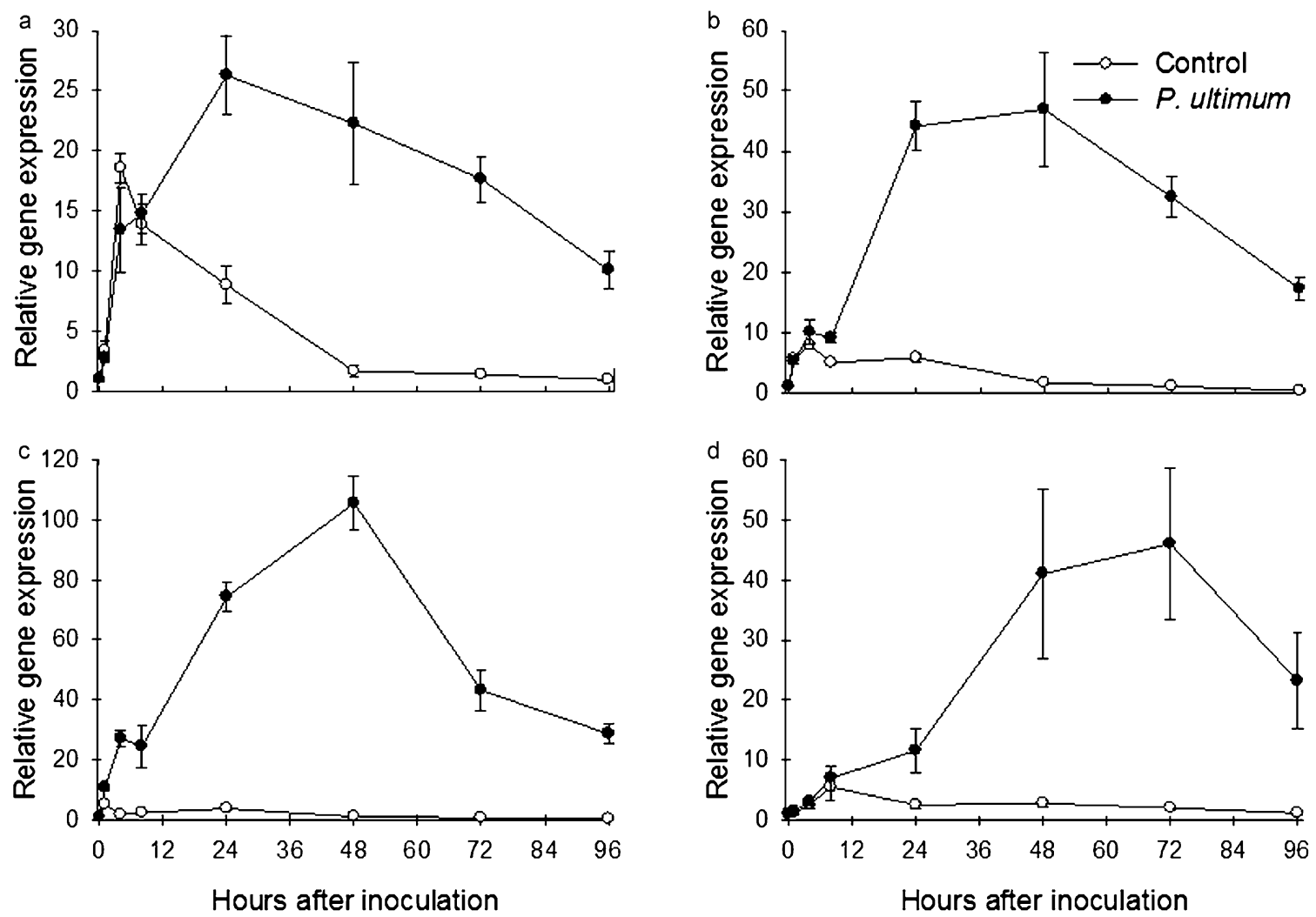

Figure 4. Gene expression profiles of three apple MdERFs and MdCHIB in response to $P$. ultimum in apple root: (a) MdERF1 (MDP0000235313); (b) MdERF1 (MDP0000127134); (c) MdERF1 (MDP0000880063); (d) MdCHIB (MDP0000430546). Each data point represents the means with standard error.

the MdACS genes (MDP0000130748 and MDP0000262827), ET acted as a negative regulator while $\mathrm{JA}$ acted as a positive regulator of their expression (Figure 7a-7d). This antagonistic regulation between the two hormones was observed in both root and shoot tissues (Figure 7a-7d). The expression of the third MdACS (MDP0000435100) was upregulated individually by ET or JA, and two hormones acted synergistically, also its expression was much higher in root than in shoot tissues (Figure 7e-7f). In response to ET and JA treatments, the expression of MdAOS (MDP0000132456) showed significantly greater upregulation in roots (approximately 70-fold increase at transcript abundance) than in shoots, but a synergistic response was only observed in shoot tissue (Figure $7 \mathrm{~g}-7 \mathrm{~h}$ ). These results added evidence that the expression of $P$. ultimum response genes is directly or indirectly regulated by both ET and JA hormonal signals.

\section{DISCUSSION}

The role of ET/JA mediated defense response in plant root, especially in perennial tree species, is still mostly unknown, ${ }^{29,30}$ though ET/JA signaling pathways in a plant defense response in leaf tissues against necrotrophic pathogens has been well established, mostly from foliar disease systems of model plants such as Arabidopsis and tobacco. ${ }^{8,31-33}$ Findings from the current study demonstrate that the ET/JA signaling pathways are functional in apple root in response to the $P$. ultimum infection. Our data showed that infection by $P$. ultimum activated ET/JA signaling-related genes, including MdACS (ethylene biosynthesis gene), MdAOS (JA biosynthesis gene), MdERF (a transcription factor gene that responds to both ET/JA hormonal signals) and MdCHIB (CHITINASEB is a target of $E R F)$ in the apple root system.
Although the transcriptional regulation of genes for ET and JA biosynthesis and signaling is just one layer of their function, results from this study provide the essential information for further elucidation of their regulating roles in defense response. The coordinated regulation patterns of genes in both ET and JA biosynthesis pathways in response to $P$. ultimum inoculation suggested that both hormones are required to activate an effective defense reaction in root tissues. Our data indicated that most ET/ JA biosynthesis genes, except MdACS (MDP0000435100), exhibited peak induction at $48 \mathrm{hpi}$. Two out of three MdERFs also showed peak expression at $48 \mathrm{hpi}$, while its downstream PR protein encoding gene $M d C H I B$ displayed maximum expression at $72 \mathrm{hpi}$. The coordinately upregulated expression of the genes suggested that a functional ET/JA-mediated defense pathway (from ET/JA biosynthesis and signaling transduction to antimicrobial activity) exists in apple roots.

Cross-talk among hormone signaling networks during plant defense has been well elucidated in which ET and JA signaling pathways regulate and modulate each other to produce effective defense responses. ${ }^{8-10,34,35}$ ET and JA independently activate not just separate sets of genes, they also synergistically activate $P R$ genes (including PDF1.2 and CHIB) through ERF in response to necrotrophic pathogens. ${ }^{6,8,34,35}$ In the JA signaling pathway, COI1 (JA receptor) receives the JA signal and initiates degradation of JAZ (JA response repressor) through ubiquitination and proteasome activity. $^{36}$ The removal of JAZ boosts EIN3/EIL1 activity which causes induction of ERF and consequently induces $C H I B$. Ethylene signaling is incorporated through enhancing EIN3/EIL1 stability. Since EIN3/EIL1 protein has a short-life cycle, the ethylene signal is essential for its build-up in the nucleus. ${ }^{37,38}$ Two MdACSs 
NDP0 000127134 NDP0 000235313 At3g 23240 VDP0 000880063 At1g 04370

VDPO000127134 NDP0 000235313 At $3 g 23240$ MDP0 000880063 At1g04370

MDP0 000127134 NDP0000235313 At3g 23240 NDP0 000880063 At1g04370

MDP0000127134 NDP0 000235313 At 3 g 23240 NDP0 000880063 At1g04370

MDP0 000127134 NDP0 000235313 At3g 23240 NDP0 000880063 At1g 04370
MACHKYTNTXXVEQAENKNTPI LOMYYTED SPNSNLSPESSFGSSESWDDGLKFGNSL 60 -

- - - - - - - -

PFIENDSE EML LYGLISEGTOEITSSVSVEANPIKEEEVSSASEEENPKKKKSYRGVRRR 120 PENENDSE EMLLYGLISEATQEITSSEFVSANPIKEEEVSSACEEENLKKEKS YRGVRRR 96 PENENDSE EYTLYGLIEQSTOQTYIDSDSQD LPIKS--VSSR--.---KSEKSYRGVRRR 88

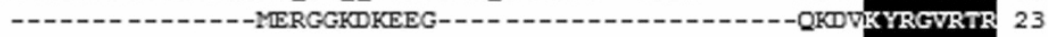
-

PWGKFAAEIRDSTRHGIRVWLGTDSABARALAYDOARFSERGSAAVLRFPVER VRKSLR 180 PWGKPAAEIRDSTRHGIRWWLGTPDSABAAALAYDOA,AFSMTRSYAILNFPVERVRESLR 156

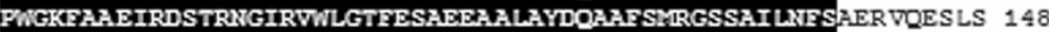

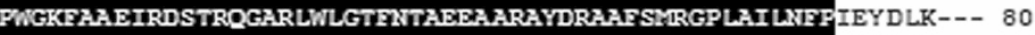

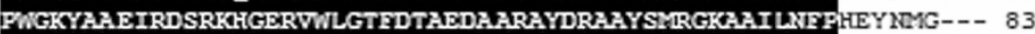

XINYGTSD LEGCSPVVALKRKHSIRRKTGNTKSK----VDRDVRIENVVVEEDLGIDYLE 236 EINYGTLD LEGCSPVIALKRKHSMRRKTGEKKSQ----VDRDVMIENVVVEEDLGTDYLE 212 EIKY--TYEDGCSPVVALKRKHSMIRRRMTNKKTKDSDFDHRSVKLDNVVVEED LGEQYLE 206 ------DADQSAAAVSSSSSSLSSSSR PHVV-----TRTESGREIFEFEC LDDSVLE 126 -------TGSSSTARNSSSSS----------------QQVEEEEY LDDSVLD 112

ELLNSSESTSTNATPNW---- 253

ELLNSTENTSTSATPWW---- 229

ELLGSSENSGT-----W---- 218

DLLDE-DNHTKTIEEKQIK-- 144

ELLEYGENYIKTHNINOMGKRQ 133

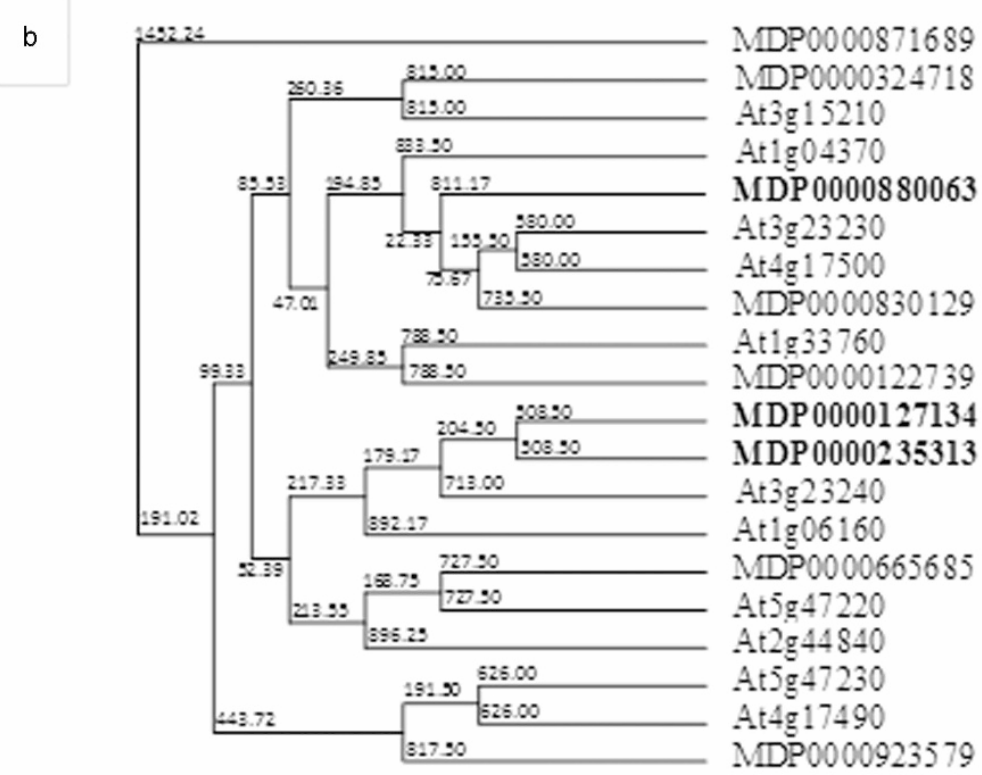

Figure 5. Sequence similarity shared between MdERFs and AtERFs. (a) Alignment of deduced amino-acid sequence of two Arabidopsis AtERFs (At3g23240 and At1g04370) and three apple MdERFs (MDP0000127134, MDP0000235313 and MDP0000880063). AP2/ERF domain is indicated by reverse lettering. (b) Phylogenetic analysis of MdERFs from this study and AtERFs that were reported as necrotrophic pathogen and JAinduced genes. Three MdERFs that were upregulated by $P$. ultimum infection are in bold.

(MDP0000130748 and MDP0000262827) and MdAOSs (only in root) were shown to be induced by JA and repressed by ET application and an antagonistic regulation by combinational ET/JA treatments. On the other hand, the expression of MdERF and MdCHIB, were upregulated by both individual ET and JA treatments or synergistically by ET/JA treatment in combination. These observations suggest that first, complicated feedback regulation existed at ET/JA biosynthesis and second, both hormones act synergistically for the signal transduction through MdERF and downstream defense actions in both apple root and shoot tissues.

For the regulation of ET/JA biosynthesis genes, our results based on exogenous ET and JA treatment assays indicated that JA always acts as a positive regulator, while ET acts as either positive or negative feedback signals. The contrasting action of ET in shoots and 

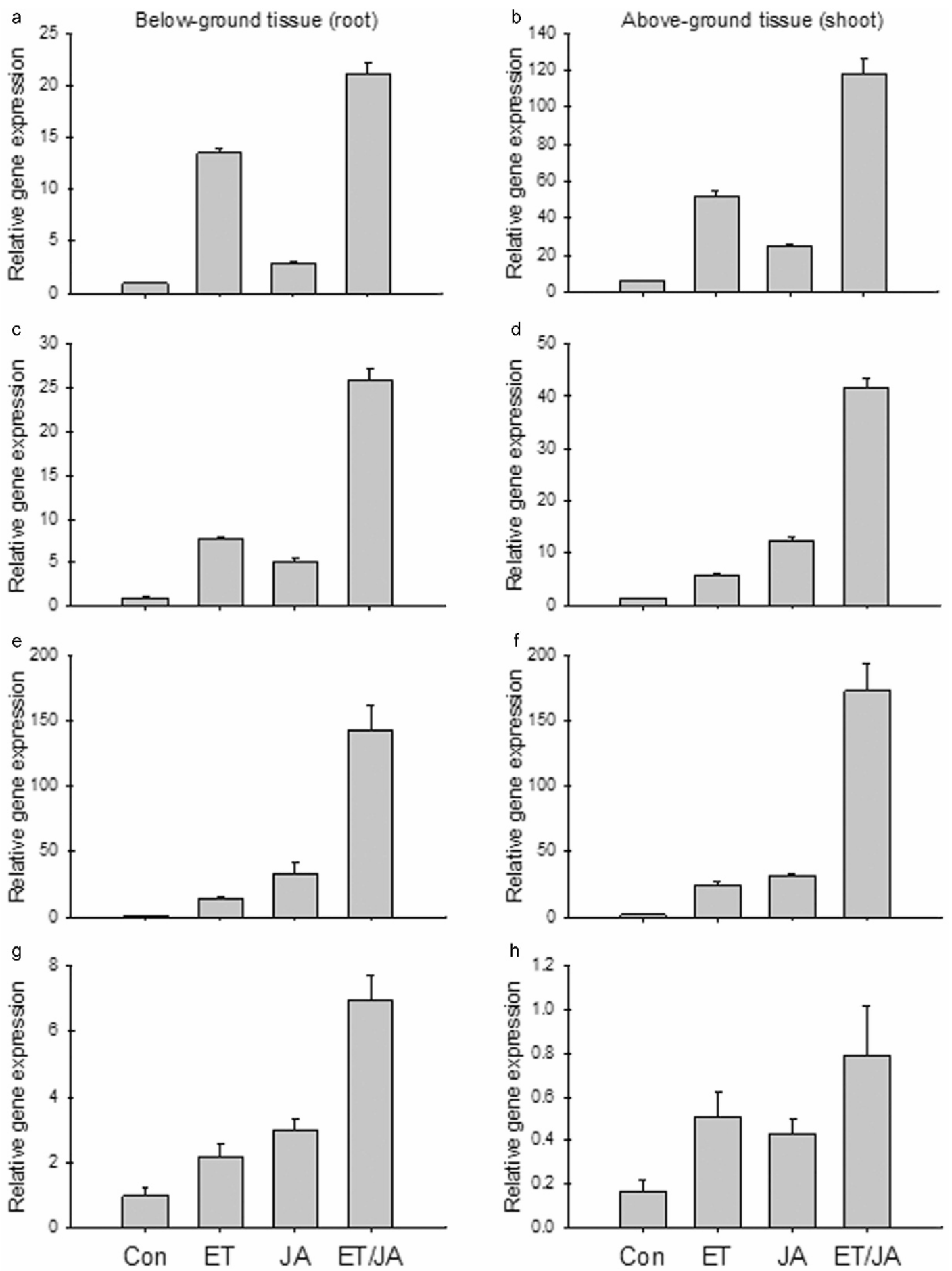

Figure 6. Gene expression profile of two apple MdERF1s with ET and JA treatment: (a and b) MdERF1 (MDP0000235313); (c and d) MdERF1 (MDP0000127134); (e and f) MdERF (MDP0000880063); (g and h) MdCHIB (MDP0000430546); (a, c, e and g) under-ground tissue (roots); (b, d, f and h) above-ground tissue (shoot). ET: 110 ppm ethylene only; JA: $500 \mu \mathrm{M}$ methyl jasmonate only; ET/JA: ethylene and methyl jasmonate together. Each data point represents the means with standard error.

roots could be due to other factors such as $\mathrm{Ca}^{2+}$ signaling and mitogen-activated protein kinase, which affect ET-mediated transcriptional regulation in addition to the major regulatory mechanism of ubiqutin-mediated protein degradation. ${ }^{15,37}$ For JA, it has been known that JA biosynthesis genes, including LOX (Lipoxygenase), AOS, AOC (Allene Oxide Cyclase), OPR3 (OPDA reductase 3) and $A C X$ (Acyl-CoA Oxidase), can be activated through the SCF ${ }^{\mathrm{COI}}$-JAZ-mediated positive feedback loop. ${ }^{15,39}$ Between root and shoot tissues, very similar gene expression patterns were observed for all genes studied except MdAOS, suggesting the function of similar molecular components in the defense response in both shoot and root tissues. 

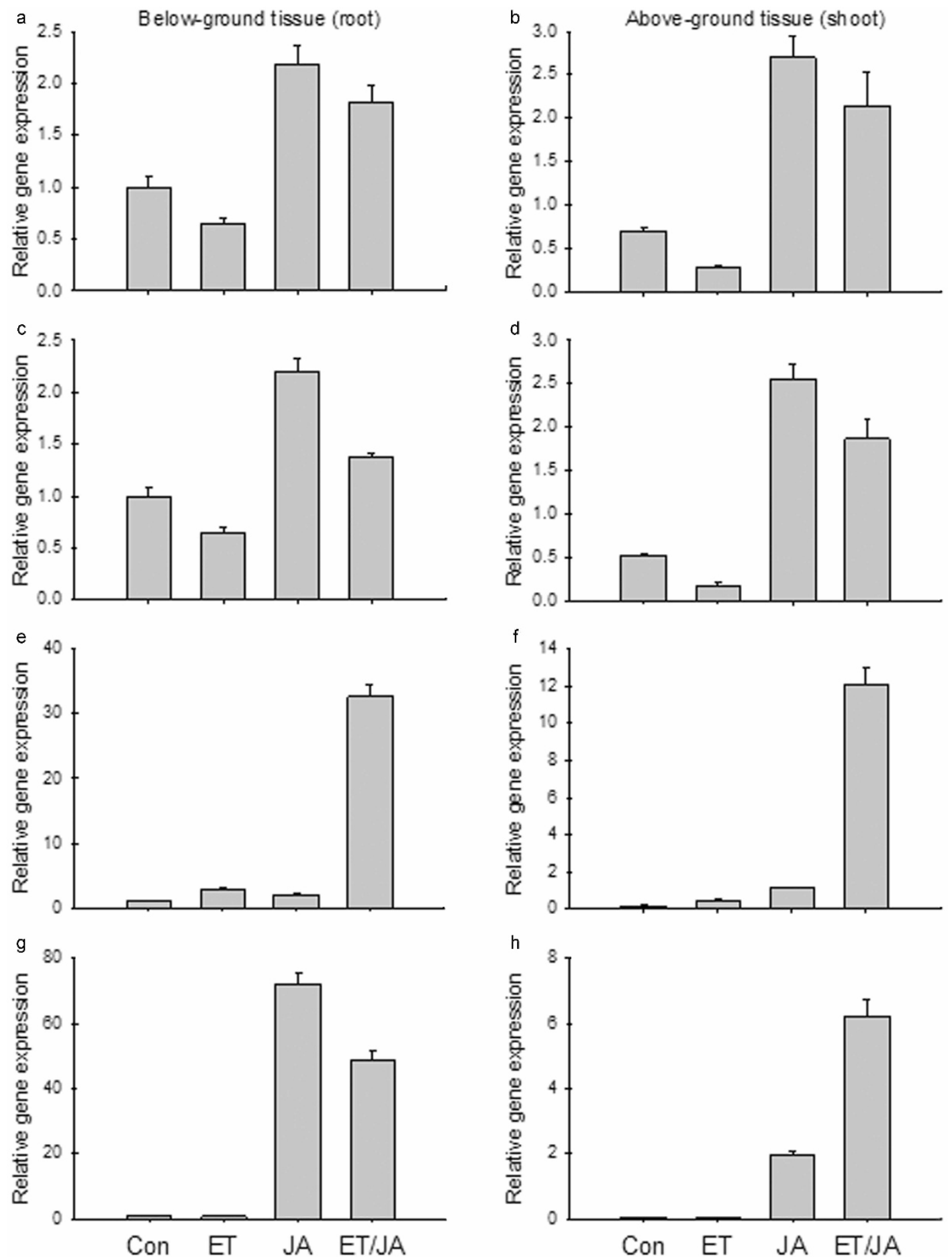

Figure 7. Gene expression profile of three apple MdACSs with ET and JA treatment: (a and b) MdACS (MDP0000130748); (c and d) MdACS (MDP0000262827); (e and f) MdACS (MDP0000435100); (g and h) MdAOS (MDP0000132456); (a, c, e and g) under-ground tissue (roots); (b, d, f and $\mathbf{h}$ ) above-ground tissue (shoot). ET: 110 ppm ethylene only; JA: $500 \mu \mathrm{M}$ methyl jasmonate only; ET/JA: ethylene and methyl jasmonate together. Each data point represents the means with standard error.

A simplified model of ET/JA biosynthesis and signaling in apple roots in response to $P$. ultimum infection was shown in Figure 8 based on the observations from this study. Infection by $P$. ultimum triggers ET/JA biosynthesis by activating MdACS and MdAOS in the apple root. ET/JA signaling pathways are activated when the
ET/JA hormonal receptor perceives signals, which synergistically upregulate the expression of MdERF1 preassembly through $\mathrm{SCF}^{\mathrm{CO} I 1}-\mathrm{JAZ}$ proteasome activity and EIN3/EIL1 transcriptional activity. MdERF1, as a transcription factor, activates the expression of $P R$ genes such as $M d C H I B$ by binding to their promoter 


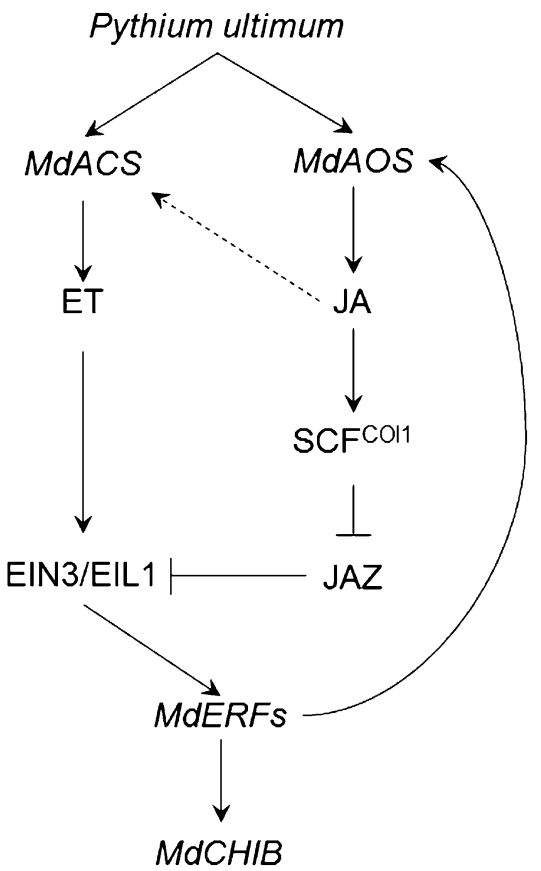

Figure 8. The proposed model of hormonal regulation (by ET and JA) on transcription of $P$. ultimum response genes in apple root. Accumulated ET and JA in response to the $P$. ultimum infection activate the MdERF1 expression through $\mathrm{SCF}^{\mathrm{CO} 11}$-JAZ proteasome activity for subsequent activation of $M d C H I B$. ET and JA also can regulate their own biosynthesis pathways as either positive or negative feedback regulation.

region. JA not only activates defense-related genes, but JA is also involved in feedback regulation of JA biosynthesis by acting as a positive regulator in the apple root system. This study serves as a proof of concept that the knowledge of plant defense response to necrotrophs, which were primarily elucidated using foliar pathogens of Arabidopsis, seems to be transferable to the interaction between root of perennial crops and soil borne necrotrophic pathogens.

\section{COMPETING INTERESTS}

The authors declare no conflicts of interest.

\section{ACKNOWLEDGEMENTS}

We thank Steve Reymond, Janie Countryman, Sheila Ivanov, Dave Buchanan and Edward Valdez for their excellent technical assistance.

\section{REFERENCES}

1 Mazzola M. Elucidation of the microbial complex having a causal role in the development of apple replant disease in Washington. Phytopathology 1998; 88: 930-938.

2 Tewoldemedhin YT, Mazzola M, Botha WJ, Spies CF, McLeod A. Characterization of fungi (Fusarium and Rhizoctonia) and oomycetes (Phytophthora and Pythium) associated with apple orchards in South Africa. Eur J Plant Pathol 2011; 130: 215-229.

3 Zhu Y, Fazio G, Mazzola M. Elucidating the molecular responses of apple rootstock resistant to ARD pathogens: challenges and opportunities for development of genomics-assisted breeding tools. Hort Res 2014; 1: 14043.

4 Li C, Shao J, Wang Y, Li W, Guo D et al. Analysis of banana transcriptome and global gene expression profiles in banana roots in response to infection by race 1 and tropical race 4 of Fusarium oxysporum f. sp. cubense. BMC Genomics 2013; 14: 851.
5 Millet YA, Danna CH, Clay NK, Songnuan W, Simon MD et al. Innate immune responses activated in Arabidopsis roots by microbe-associated molecular patterns. Plant Cell 2010; 22: 973-990.

6 Shinshi $\mathrm{H}$. Ethylene-regulated transcription and crosstalk with jasmonic acid. Plant Sci 2008; 175: 18-23.

7 Kazan K, Manners JM. Jasmonate signaling: toward an integrated view. Plant Physiol 2008; 146: 1459-1468.

8 Okubara P, Paulitz T. Root defense responses to fungal pathogens: a molecular perspective. Plant Soil 2005; 274: 215-226.

9 Glazebrook J. Contrasting mechanisms of defense against biotrophic and necrotrophic pathogens. Annu Rev Phytopathol 2005; 43: 205-227.

10 Pieterse CM, van der Does D, Zamioudis C, Leon-Reyes A, van Wees SC. Hormonal modulation of plant immunity. Annu Rev Cell Dev Biol 2012; 28: 489-521.

11 Ballare CL. Jasmonate-induced defenses: a tale of intelligence, collaborators and rascals. Trends Plant Sci 2011; 16: 249-257.

12 Robert-Seilaniantz A, Grant M, Jones JD. Hormone crosstalk in plant disease and defense: more than just jasmonate-salicylate antagonism. Annu Rev Phytopathol 2011; 49: 317-343.

13 Acosta IF, Farmer EE. Jasmonates. The Arabidopsis Book 2010; 8: e0129.

14 Mengiste T. Plant immunity to necrotrophs. Annu Rev Phytopathol 2012; 50: 267294.

15 Wasternack C, Hause B. Jasmonates: biosynthesis, perception, signal transduction and action in plant stress response, growth and development. An update to the 2007 review in Annals of Botany. Ann Bot 2013; 111: 10211058.

16 Solano R, Stepanova A, Chao Q, Ecker JR. Nuclear events in ethylene signaling: a transcriptional cascade mediated by ETHYLENE-INSENSITIVE3 and ETHYLENERESPONSE-FACTOR1. Genes Dev 1998; 12: 3703-3714.

17 Berrocal-Lobo M, Molina A, Solano R. Constitutive expression of ETHYLENERESPONSE-FACTOR1 in Arabidopsis confers resistance to several necrotrophic fungi. Plant J 2002; 29: 23-32.

18 Wasternack C. Jasmonates: an update on biosynthesis, signal transduction and action in plant stress response, growth and development. Ann Bot 2007; 100: 681-697.

19 Lorenzo O, Piqueras R, Sánchez-Serrano JJ, Solano R. ETHYLENE RESPONSE FACTOR1 integrates signals from ethylene and jasmonate pathways in plant defense. Plant Cell 2003; 15: 165-178.

20 Velasco R, Zharkikh A, Affourtit J, Dhingra A, Cestaro A et al. The genome of the domesticated apple (Malus $x$ domestica Borkh.). Nat Genet 2010; 42: 833839.

21 Fazio G, Aldwinckle HS, Robinson TL, Cummins J. (314) Geneva ${ }^{\circledR}$ 41: a new fire blight resistant, dwarf apple rootstock. HortScience 2005; 40: 1027.

22 Wertheim SJ. Rootstock Guide: Apple, Pear, Cherry, European Plum. Wilhelminadorp: Fruit Research Station; 1998.

23 Gasic K, Hernandez A, Korban S. RNA extraction from different apple tissues rich in polyphenols and polysaccharides for cDNA library construction. Plant Mol Biol Rep 2004; 22: 437-438.

24 Livak KJ, Schmittgen TD. Analysis of relative gene expression data using real-time quantitative PCR and the $2^{-\Delta \Delta C T}$ method. Methods 2001; 25: 402-408.

25 Schmittgen TD, Livak KJ. Analyzing real-time PCR data by the comparative $C_{T}$ method. Nat Protoc 2008; 3: 1101-1108.

26 McGrath KC, Dombrecht B, Manners JM, Schenk PM, Edgar Cl et al. Repressorand activator-type ethylene response factors functioning in jasmonate signaling and disease resistance identified via a genome-wide screen of Arabidopsis transcription factor gene expression. Plant Physiol 2005; 139: 949-959.

27 Okamuro JK, Caster B, Villarroel R, van Montagu M, Jofuku KD. The AP2 domain of APETALA2 defines a large new family of DNA binding proteins in Arabidopsis. Proc Natl Acad Sci USA 1997; 94: 7076-7081.

28 Pre M, Atallah M, Champion A, de Vos M, Pieterse CM et al. The AP2/ERF domain transcription factor ORA59 integrates jasmonic acid and ethylene signals in plant defense. Plant Physiol 2008; 147: 1347-1357.

29 Vijayan P, Shockey J, Lévesque CA, Cook RJ, Browse J. A role for jasmonate in pathogen defense of Arabidopsis. Proc Natl Acad Sci USA 1998; 95: 72097214.

30 Geraats BP, Bakker PA, van Loon LC. Ethylene insensitivity impairs resistance to soilborne pathogens in tobacco and Arabidopsis thaliana. Mol Plant Microbe Interact 2002; 15: 1078-1085.

31 Wang KL, Li H, Ecker JR. Ethylene biosynthesis and signaling networks. Plant Cell 2002; 14(Suppl 1): S131-S151.

32 Turner JG, Ellis C, Devoto A. The jasmonate signal pathway. Plant Cell 2002; 14(Suppl 1): S153-S164.

33 Broekaert WF, Delauré SL, de Bolle MF, Cammue BP. The role of ethylene in hostpathogen interactions. Annu Rev Phytopathol 2006; 44: 393-416. 
34 Dong X. SA, JA, ethylene, and disease resistance in plants. Curr Opin Plant Biol 1998; 1: 316-323.

35 Feussner I, Wasternack C. The lipoxygenase pathway. Annu Rev Plant Biol 2002; 53 : 275-297.

36 Wager A, Browse J. Social network: JAZ protein interactions expand our knowledge of jasmonate signaling. Front Plant Sci 2012; 3: 41.

37 Zhao Q, Guo HW. Paradigms and paradox in the ethylene signaling pathway and interaction network. Mol Plant 2011; 4: 626-634.

38 An F, Zhao Q, Ji Y, Li W, Jiang Z et al. Ethylene-induced stabilization of ETHYLENE INSENSITIVE3 and EIN3-LIKE1 is mediated by proteasomal degradation of EIN3 binding F-box 1 and 2 that requires EIN2 in Arabidopsis. Plant Cell 2010; 22 2384-2401.
39 Chung HS, Koo AJ, Gao X, Jayanty S, Thines B et al. Regulation and function of Arabidopsis JASMONATE ZIM-domain genes in response to wounding and herbivory. Plant Physiol 2008; 146: 952-964.

(c) (1) (-) $\odot$ This work is licensed under a Creative Commons Attribution-

cc) NonCommercial-NoDerivs 3.0 Unported License. The images or other third party material in this article are included in the article's Creative Commons license, unless indicated otherwise in the credit line; if the material is not included under the Creative Commons license, users will need to obtain permission from the license holder to reproduce the material. To view a copy of this license, visit http:// creativecommons.org/licenses/by-nc-nd/3.0/

Supplemental Information for this article can be found on the Horticulture Research website (http://www.nature.com/hortres). 